\title{
Ocorrência de quedas em idosos não institucionalizados: revisão sistemática da literatura
}

\author{
Falls in the non-institutionalized elderly: a systematic literature review
}

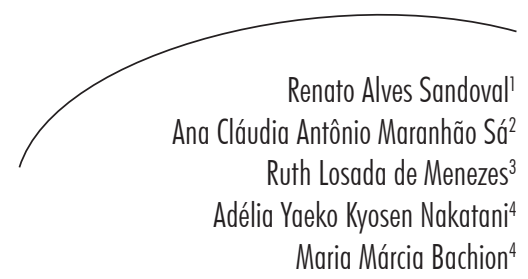

\section{Resumo}

Revisão sistemática da literatura com o objetivo de identificar a ocorrência de quedas de idosos residentes na comunidade. Utilizando o portal CAPES, realizou-se busca nas bases de dados Academic Search (EBSCO), SciELO e Science Direct (ELSEVIER); e uma busca adicional na base de dados Lecturas Educación Física y Deportes, do site EFDEPORTES. Foram encontrados, com os descritores utilizados, 247 artigos publicados no período de 2000 a 2011. Foram excluídos 230 que não atenderam a um ou mais dos critérios de inclusão. Incluíram-se no estudo 17 artigos, sendo 12 transversais, dois de coorte prospectiva, dois de pesquisa de base populacional e um de coorte retrospectiva. A amostra total envolvida nos estudos foi de 114.911 sujeitos, com média de idade de aproximadamente 70 anos, distribuídos em sete países: Brasil, Estados Unidos, Espanha, Itália, Nigéria, Turquia e China. A ocorrência de quedas variou de 15,9 a 56,3\%, com mediana de 28,50\%. Embora apresente variação, a ocorrência de quedas na população idosa que reside na comunidade é alta.

\section{Abstract}

This systematic literature review aimed to identify the occurrence of falls in elderly living in the community. On CAPES website, a search was conducted on the following databases: Academic Search (EBSCO), SciELO, and Science Direct (ELSEVIER). An additional search, on the EFDEPORTES website, was performed on the Lecturas Educación Física y Deportes database. Using the chosen descriptors, 247 articles published from 2000 to 2011 were identified. A total of 230 articles were excluded as they did not meet all the inclusion criteria. Hence, 17 articles were included in the study, 12 of

Palavras-chave: Acidente por Quedas. Síncope. Idoso. Epidemiologia.
Key words: Accidental Falls. Syncope. Elderly. Epidemiology.

\footnotetext{
Departamento de Enfermagem, Nutrição, Fisioterapia e Gastronomia, Campus I, área VI, Unidade Goiânia. Pontifícia Universidade Católica de Goiás. Goiânia, GO, Brasil.

2 Departamento de Fisioterapia, Faculdade de Fisioterapia, Campus Jataí. Universidade Federal de Goiás. Jataí, GO, Brasil.

3 Programa de Pós-graduação em Ciências e Tecnologia em Saúde. Universidade de Brasília. Brasília, DF, Brasil.

4 Departamento de Enfermagem, Faculdade de Enfermagem. Universidade Federal de Goiás. Goiânia, GO, Brasil.
} 
which were cross-sectional studies, two prospective studies, two population studies, and one retrospective study. The whole sample involved in the studies consisted of 114,911 subjects with an average age of 70 years, distributed across seven countries: Brazil, United States, Spain, Italy, Nigeria, Turkey and China. The occurrence of falls ranged between $15.9 \%$ and $56.3 \%$, with a median of $28.50 \%$. Although presenting variation, the occurrence of falls in elderly living in the community is still high.

\section{INTRODUÇÃO}

Pesquisas apontam quatro grandes síndromes relacionadas ao envelhecimento, as quais necessitam de cuidados específicos. São elas: incontinência urinária, demência, imobilidade e quedas. Esta última pode ser definida como um evento involuntário, que precipita a pessoa ao solo ou a um nível inferior do qual se encontrava anteriormente. ${ }^{1,2}$

A prevalência de quedas referida por estudiosos da área é de aproximadamente 30\%, tanto em idosos na comunidade como residentes em instituições de longa permanência; entre os idosos que sofreram uma queda, estima-se que $50 \%$ terão novos episódios, cerca de $10 \%$ apresentarão fraturas e mais de $20 \%$ diminuirão suas atividades por medo de cair novamente..$^{3-8}$

Com o avanço da idade, os episódios de queda se tornam mais frequentes; na pessoa muito idosa, há controvérsias entre os achados, uma vez que alguns estudos apontam a diminuição da frequência de quedas nesse grupo, provavelmente devido à diminuição da mobilidade, e outros mostram o aumento do número de quedas devido à fragilidade. ${ }^{4,9}$

A etiologia das quedas é multifatorial e está relacionada a fatores intrínsecos como transtornos da visão, do equilíbrio, da marcha, e fatores extrínsecos, como riscos ambientais. Os fatores intrínsecos são responsáveis pelo maior número de quedas em idosos institucionalizados; já os fatores extrínsecos são os responsáveis por quedas em idosos da comunidade. , $^{8,10}$

Pela importância desse evento na saúde dos idosos, estudos sobre a temática, em diferentes regiões geográficas, seriam relevantes. Considerando que a ocorrência de quedas e a magnitude de seus fatores de risco podem variar conforme as condições de vida da população, com impacto no processo de envelhecimento, emerge a questão norteadora deste estudo: qual é a ocorrência de quedas em diferentes regiões geográficas no Brasil e no mundo?

Para responder a essa pergunta, desenvolveuse o presente estudo, com o objetivo de identificar os conhecimentos científicos publicados sobre a prevalência de quedas em população de idosos residentes na comunidade.

\section{MATERIAIS E MÉTODOS}

\section{Estratégia de busca}

Trata-se de revisão sistemática da literatura, realizada nas seguintes bases de dados: Academic Search (EBSCO), SciELO e Science Direct (ELSEVIER), acessadas por meio do Portal CAPES de periódicos, e Lecturas Educación Física y Deportes, no site EFDEPORTES. Os descritores utilizados foram: em português (quedas e idosos), em inglês (falls and elderly) e em espanhol (las caídas $y$ personas mayores o adulto mayor o ancianos).

\section{Seleção dos estudos}

Foram selecionados para este estudo artigos que atenderam aos seguintes critérios de inclusão: constituir estudo primário publicado em português, inglês ou espanhol, no período de 2000 a 2011; ter como amostra idosos da comunidade; e como desfecho, a ocorrência de quedas nesta população. 
Mediante utilização das palavras-chave, considerando todas as bases, foram recuperados 247 artigos. Numa segunda etapa do processo de seleção dos artigos, os títulos e resumos foram analisados por dois pesquisadores, em relação aos critérios de inclusão, de modo independente. Foram excluídos 198 artigos, em virtude de estarem publicados em outros idiomas que não fossem inglês, português e espanhol e/ou serem publicados em período anterior a $2000 \mathrm{e} / \mathrm{ou}$ não incluírem na amostra idosos da comunidade e/ ou não constituírem estudos primários. Assim, foram selecionados 49 estudos que atenderam aos critérios de seleção estabelecidos para a pesquisa.

\section{Análise dos artigos}

Os 49 artigos incluídos nessa etapa foram analisados na íntegra, em relação à qualidade do delineamento, consistência dos procedimentos adotados para a coleta de dados e constituição da amostra de idosos em geral.

Foram excluídos 32 artigos, por uma ou mais das seguintes razões: dez não abordavam a queda como desfecho; oito não definiram o método utilizado para verificar a ocorrência de quedas; quatro não apresentaram procedimentos para amostragem; quatro continham pessoas com menos de 60 anos de idade na amostra; e ainda seis estudos abordavam grupos específicos de idosos da comunidade (mulheres, idosos com fragilidade, idosos da zona rural, entre outros, e usuários de determinados serviços de saúde). Assim, foram incluídos no estudo 17 artigos $(34,69 \%)$.

\section{Descrição dos artigos}

Realizou-se uma síntese das informações de cada estudo em um quadro contendo os seguintes tópicos: autor(es) e ano; local do estudo (cidade, país); delineamento; objetivos; amostra e faixa etária (média e desvio-padrão); e definição e ocorrência de quedas.

\section{RESULTADOS}

Conforme se pode verificar nas pesquisas selecionadas para análise, descritas no quadro 1, os cenários dos estudos de ocorrência de quedas em idosos no período analisado estão distribuídos em sete países: América do Sul Brasil (7 artigos); Europa - Espanha (3 artigos) e Itália (1 artigo); África - Nigéria (1 artigo); Oriente Médio - Turquia (2 artigos); Ásia China (2 artigos); e América do Norte - Estados Unidos (1 artigo).

É oportuno esclarecer que não foram encontradas, nas bases de dados consultadas, outras pesquisas realizadas em países da América do Norte que pudessem ser incluídas considerando os critérios estabelecidos descritos. Nessa região, os estudos identificados envolveram idosos atendidos em serviços de saúde, idosos atendidos em decorrência de morbidades relacionadas a quedas e grupos específicos de idosos da comunidade.

Em relação ao delineamento dos estudos, 12 $(70,5 \%)$ eram transversais; dois $(11,7 \%)$ de coorte prospectiva; dois $(11,7 \%)$ de pesquisa de base populacional e um $(6,1 \%)$ de coorte retrospectiva. A maior parte dos estudos apresentou como objetivo identificar a prevalência e/ou incidência de quedas e os fatores associados e, em alguns estudos, cujo objetivo principal não se referia à ocorrência de queda, esse dado foi apresentado no bojo dos resultados, como desfecho.

Em relação ao tamanho da amostra dos estudos, a menor foi de 70 idosos e a maior amostra continha 92.808 idosos. O somatório de todas as amostras analisadas totalizou 114.911 sujeitos.

Quando analisada a ocorrência de quedas encontrou-se, no geral, uma variação entre 15,9\% e $56,3 \%$, e em ambos os casos a média de idade dos idosos foi de 70 anos. A menor prevalência foi encontrada nos Estados Unidos, e a maior, no Brasil. Na Europa, estudos realizados na Espanha e Itália mostraram prevalência de 
$30,5 \%$ a $31,8 \%$. Na África, o estudo realizado na Nigéria mostrou a prevalência de $23 \%$. Na Ásia, o estudo realizado na China evidenciou prevalência de $26,4 \%$.

No Brasil foi encontrado o maior número de estudos de prevalência de quedas em idosos, sendo que as regiões mais estudadas foram a Sul, Sudeste e Nordeste. Apenas um estudo inclui dados relativos à Região Norte. O segundo país em número de estudos identificados foi Espanha, com dados provenientes de duas cidades.
Os únicos países que dispõem de dados da população em toda sua extensão, publicados no período analisado, foram Brasil, Estados Unidos e Nigéria.

Foram identificados apenas três estudos de coorte, sendo dois prospectivos, realizados na cidade de Barcelona, Espanha, nos quais se encontraram 26,4 e 36,0\% para prevalência de quedas em idosos da comunidade. Um estudo de coorte, retrospectivo, foi realizado em Hong Kong, China, encontrando valor de $20 \%$ de incidência de quedas.

Quadro 1 - Análise dos artigos incluídos com ocorrência de quedas como variável dependente ( $\mathrm{n}=17)$, 2000-2011. ${ }^{4-6,11-24}$

\begin{tabular}{|c|c|c|c|c|c|}
\hline Autores & Local & $\begin{array}{c}\text { Delineamento, } \\
\text { variável, } \\
\text { período }\end{array}$ & Objetivos & $\begin{array}{l}\text { Amostra(n) } \\
\text { e faixa etária } \\
(m \pm d p)\end{array}$ & $\begin{array}{c}\text { Ocorrência } \\
\text { de quedas } \\
(\%)\end{array}$ \\
\hline $\begin{array}{l}\text { Moreno-Martínez } \\
\text { et al. } 2005^{4}\end{array}$ & $\begin{array}{l}\text { Barcelona, } \\
\text { Espanha }\end{array}$ & $\begin{array}{l}\text { Coorte } \\
\text { prospectiva, } \\
\text { incidência, } 12 \\
\text { meses }\end{array}$ & $\begin{array}{l}\text { Desenvolver um } \\
\text { programa de } \\
\text { prevenção de quedas }\end{array}$ & $\begin{array}{c}200 \\
79,5 \pm 3,6\end{array}$ & 36,0 \\
\hline $\begin{array}{l}\text { Varas-Fabra et al. } \\
2006^{5}\end{array}$ & $\begin{array}{l}\text { Córdoba, } \\
\text { Espanha }\end{array}$ & $\begin{array}{l}\text { Transversal, } \\
\text { prevalência, } 12 \\
\text { meses }\end{array}$ & $\begin{array}{l}\text { Descrever a } \\
\text { prevalência de quedas }\end{array}$ & $\begin{array}{c}362 \\
76,6 \pm 5,37\end{array}$ & 31,8 \\
\hline $\begin{array}{l}\text { Formiga et al. } \\
2008^{11}\end{array}$ & $\begin{array}{l}\text { Barcelona, } \\
\text { Espanha }\end{array}$ & $\begin{array}{l}\text { Coorte } \\
\text { prospectiva, } \\
\text { incidência, } 12 \\
\text { meses }\end{array}$ & $\begin{array}{l}\text { Analisar a diferença } \\
\text { entre caidores e não } \\
\text { caidores }\end{array}$ & $\begin{array}{c}140 \\
93,7 \pm 2,8\end{array}$ & 26,4 \\
\hline $\begin{array}{l}\text { Monaco et al. } \\
2009^{12}\end{array}$ & Torino, Itália & $\begin{array}{l}\text { Transversal, } \\
\text { prevalência, seis } \\
\text { meses }\end{array}$ & $\begin{array}{l}\text { Descrever prevalência } \\
\text { de quedas na } \\
\text { comunidade }\end{array}$ & $\begin{array}{c}95 \\
80 \pm 6,56\end{array}$ & 30,5 \\
\hline Halil et al. $2006^{13}$ & $\begin{array}{l}\text { Ancara, } \\
\text { Turquia }\end{array}$ & $\begin{array}{l}\text { Transversal, } \\
\text { prevalência, } 34 \\
\text { meses }\end{array}$ & $\begin{array}{l}\text { Associar alterações } \\
\text { clínicas e quedas }\end{array}$ & $\begin{array}{c}2.322 \\
71,8\end{array}$ & 28,5 \\
\hline Ulus et al. $2011^{14}$ & $\begin{array}{l}\text { Ondokus, } \\
\text { Turquia }\end{array}$ & $\begin{array}{l}\text { Transversal, } \\
\text { prevalência, um } \\
\text { mês }\end{array}$ & $\begin{array}{l}\text { Determinar a } \\
\text { validade da Escala } \\
\text { Internacional de } \\
\text { Eficácia em Quedas }\end{array}$ & $\begin{array}{c}70 \\
69,7 \pm 4,59\end{array}$ & 32,9 \\
\hline $\begin{array}{l}\text { Bekibele \& } \\
\text { Gureje, 2010 }\end{array}$ & Nigéria & $\begin{array}{l}\text { Transversal, } \\
\text { prevalência, } 12 \\
\text { meses }\end{array}$ & $\begin{array}{l}\text { Determinar a } \\
\text { prevalência de quedas } \\
\text { e fatores associados na } \\
\text { população de idosos } \\
\text { da Nigéria }\end{array}$ & $\begin{array}{c}2.096 \\
75,2\end{array}$ & 23,0 \\
\hline
\end{tabular}




\begin{tabular}{|c|c|c|c|c|c|}
\hline Autores & Local & $\begin{array}{c}\text { Delineamento, } \\
\text { variável, } \\
\text { período }\end{array}$ & Objetivos & $\begin{array}{l}\text { Amostra(n) } \\
\text { e faixa etária } \\
(m \pm d p)\end{array}$ & $\begin{array}{c}\text { Ocorrência } \\
\text { de quedas } \\
(\%)\end{array}$ \\
\hline $\begin{array}{l}\text { Chu, Chiu, Chi, } \\
2008^{16}\end{array}$ & $\begin{array}{l}\text { Hong Kong, } \\
\text { China }\end{array}$ & $\begin{array}{l}\text { Pesquisa de base } \\
\text { populacional, } \\
\text { prevalência, } 12 \\
\text { meses }\end{array}$ & $\begin{array}{l}\text { Investigar o impacto } \\
\text { das quedas na } \\
\text { população }\end{array}$ & $\begin{array}{c}1.517 \\
73,2 \pm 6,3\end{array}$ & 26,4 \\
\hline Fong et al. $2011^{17}$ & $\begin{array}{l}\text { Hong Kong, } \\
\text { China }\end{array}$ & $\begin{array}{l}\text { Coorte } \\
\text { retrospectiva, } \\
\text { incidência, } \\
\text { quatro meses }\end{array}$ & $\begin{array}{l}\text { Descrever a incidência } \\
\text { e as características das } \\
\text { quedas }\end{array}$ & $\begin{array}{c}554 \\
74,8 \pm 6,7\end{array}$ & 20,0 \\
\hline $\begin{array}{l}\text { Siqueira et al. } \\
2011^{18}\end{array}$ & Brasil & $\begin{array}{l}\text { Pesquisa de base } \\
\text { populacional, } \\
\text { prevalência, } 12 \\
\text { meses }\end{array}$ & $\begin{array}{l}\text { Determinar a } \\
\text { prevalência de quedas } \\
\text { nas várias regiões do } \\
\text { Brasil }\end{array}$ & $\begin{array}{c}6.616 \\
70,9 \pm 8\end{array}$ & 27,6 \\
\hline Cruz et al. $2011^{19}$ & $\begin{array}{l}\text { São Paulo-SP, } \\
\text { Brasil }\end{array}$ & $\begin{array}{l}\text { Transversal, } \\
\text { prevalência, } 12 \\
\text { meses }\end{array}$ & $\begin{array}{l}\text { Determinar a } \\
\text { prevalência de quedas } \\
\text { em idosos com dor } \\
\text { crônica. }\end{array}$ & $\begin{array}{c}213 \\
70,8 \pm 0,7\end{array}$ & 56,3 \\
\hline $\begin{array}{l}\text { Siqueira et al. } \\
2007^{20}\end{array}$ & $\begin{array}{l}\text { Sete estados } \\
\text { das regiões } \\
\text { Sul e Nordeste } \\
\text { do Brasil }\end{array}$ & $\begin{array}{l}\text { Transversal, } \\
\text { prevalência, } 12 \\
\text { meses }\end{array}$ & $\begin{array}{l}\text { Descrever a } \\
\text { prevalência de quedas } \\
\text { e fatores associados }\end{array}$ & $\begin{array}{c}4.003 \\
73,9\end{array}$ & 34,8 \\
\hline Mazo et al. $2007^{6}$ & $\begin{array}{l}\text { São José-SC, } \\
\text { Brasil }\end{array}$ & $\begin{array}{l}\text { Transversal, } \\
\text { prevalência, três } \\
\text { meses }\end{array}$ & $\begin{array}{l}\text { Associar atividade } \\
\text { física e quedas }\end{array}$ & $\begin{array}{c}254 \\
70,9 \pm 6,7\end{array}$ & 18,9 \\
\hline $\begin{array}{l}\text { Coimbra et al. } \\
2010^{21}\end{array}$ & $\begin{array}{l}\text { Amparo-SP, } \\
\text { Brasil }\end{array}$ & $\begin{array}{l}\text { Transversal, } \\
\text { prevalência, } \\
\text { cinco meses }\end{array}$ & $\begin{array}{l}\text { Determinar os fatores } \\
\text { associados às quedas }\end{array}$ & $\begin{array}{c}2.209 \\
70,6 \pm 7,78\end{array}$ & 27,1 \\
\hline Motta et al. $2010^{22}$ & $\begin{array}{l}\text { Engenheiro } \\
\text { Paulo Frontin- } \\
\text { RJ, Brasil }\end{array}$ & $\begin{array}{l}\text { Transversal, } \\
\text { prevalência, } 12 \\
\text { meses }\end{array}$ & $\begin{array}{l}\text { Determinar a } \\
\text { prevalência de quedas } \\
\text { e fatores associados na } \\
\text { população da cidade }\end{array}$ & $\begin{array}{c}1.064 \\
71,4 \pm 8,6\end{array}$ & 30,3 \\
\hline $\begin{array}{l}\text { Nunes et al. } \\
2010^{23}\end{array}$ & $\begin{array}{l}\text { Goiânia-GO, } \\
\text { Brasil }\end{array}$ & $\begin{array}{l}\text { Transversal, } \\
\text { prevalência, } 49 \\
\text { meses }\end{array}$ & $\begin{array}{l}\text { Avaliar a capacidade } \\
\text { funcional e } \\
\text { descrever os perfis } \\
\text { socioeconômicos e } \\
\text { demográficos }\end{array}$ & $\begin{array}{c}388 \\
65 \text { anos ou } \\
\text { mais }\end{array}$ & 38,7 \\
\hline $\begin{array}{l}\text { Stevens et al. } \\
2008^{24}\end{array}$ & $\begin{array}{l}\text { Estados } \\
\text { Unidos }\end{array}$ & $\begin{array}{l}\text { Transversal, } \\
\text { prevalência, três } \\
\text { meses }\end{array}$ & $\begin{array}{l}\text { Avaliar a prevalência } \\
\text { de quedas de idosos da } \\
\text { comunidade }\end{array}$ & $\begin{array}{c}92.808 \\
65 \text { anos ou } \\
\text { mais }\end{array}$ & 15,9 \\
\hline
\end{tabular}


Os resultados da prevalência de quedas em idosos nos estudos incluídos nesta revisão se concentram nos anos 2010 e 2011 (50\% das publicações). Considerando-se todas as prevalências identificadas (figura 1), verificouse que a mediana foi de $28,50 \%$, a média de $29,71 \%$ com desvio- padrão de $9,19 \%$ e a moda foi de $26,4 \%$.

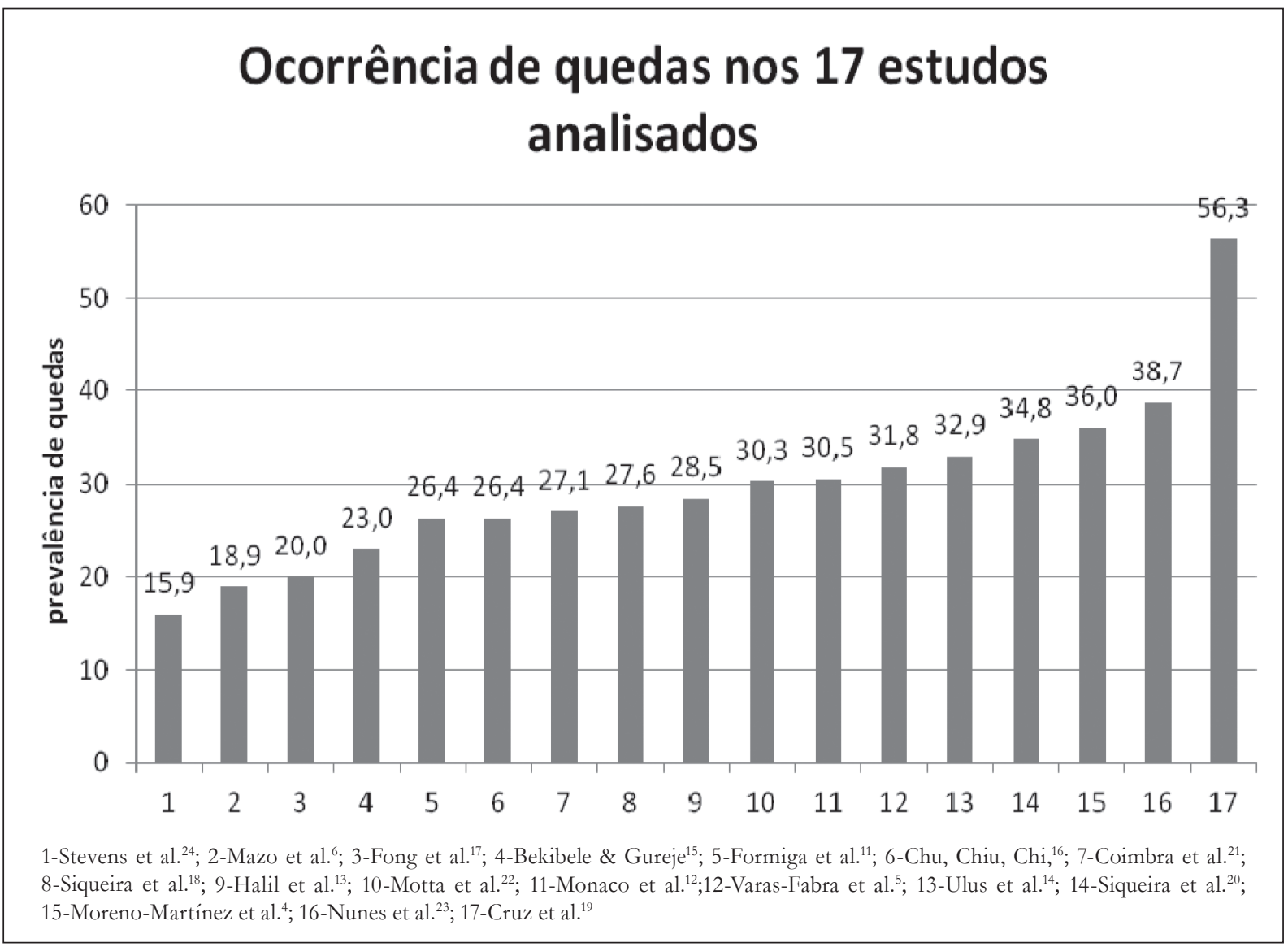

Figura 1 - Ocorrência de quedas dos estudos analisados, 2000-2011.

\section{DISCUSSÃO}

O alto número de exclusões de artigos a partir do levantamento nas bases de dados usando os descritores foi alto, devido aos vários fatores intervenientes.

Foram encontrados artigos que utilizaram, nos descritores, o termo "idosos", mas na amostra identificaram-se pessoas com menos de 60 anos de idade. Nesta revisão, utilizouse a idade de 60 anos para definir o indivíduo como idoso. Na literatura, a definição da idade do indivíduo considerado idoso apresenta variação entre 60 e 65 anos, mas estudos sobre fenômenos que dizem respeito à velhice foram desenvolvidos envolvendo grupos com idade a partir dos 50 anos, embora essa faixa etária não defina a pessoa como idosa. ${ }^{25}$

Os termos "comunidade" ou "não institucionalizado" não fazem parte dos descritores das bases pesquisadas, sendo difícil a separação dos artigos de interesse, por cenário específico. Em estudos epidemiológicos sobre temas relacionados ao envelhecimento, pode ser mais prático envolver na amostra idosos institucionalizados, por conviverem em um mesmo ambiente, favorecendo maior controle das variáveis e facilitando a coleta de dados. ${ }^{26}$ 
Em nenhum dos 17 artigos analisados encontrou-se explícito o conceito de queda adotado. Fica implícito nos trabalhos que queda é o evento que leva o indivíduo ao solo de forma involuntária. Considerando a conceituação de queda como um evento involuntário que precipita a pessoa ao solo ou a um nível inferior do qual se encontrava anteriormente, ${ }^{1,2}$ é possível supor que esse evento possa estar sendo subdetectado nas pesquisas sobre sua prevalência e incidência.

Em relação ao delineamento, o predomínio de estudos transversais se justifica por serem de mais fácil execução e permitem, da mesma forma que os delineamentos longitudinais, identificar a prevalência de quedas. Estudos de coorte demandam esforço dos pesquisadores por mais tempo no trabalho de campo. ${ }^{27}$ Nesse aspecto, outro ponto a ser discutido em relação ao delineamento é que, na maioria dos estudos, sobretudo nos transversais, os idosos relatam suas quedas. Esse método nem sempre fornece dados acurados, pois esses eventos podem não ser lembrados com facilidade, principalmente se suas consequências tiverem sido pouco significativas. Por outro lado, mesmo quando os idosos se recordam da queda sofrida anteriormente, os relatos podem não apresentar o detalhamento suficiente para uma boa investigação em relação aos fatores envolvidos.

Os valores de incidência e prevalência de quedas são empregados frequentemente na literatura de forma errônea, ${ }^{28,29}$ sendo que a falta de distinção entre os dois termos implica conclusões inadequadas para os estudos. Outro aspecto a ser considerado tanto em estudos retrospectivos quanto nos prospectivos é a heterogeneidade dos períodos de observação. Essas diferenças metodológicas entre os estudos trazem limitações para as possíveis comparações entre os mesmos.

Quanto aos cenários em que foram desenvolvidos os estudos, pode-se dizer que aqueles em que foram realizadas pesquisas de maior extensão, como Brasil e Nigéria, estão vivenciando um aumento acelerado do número de idosos que compõem suas populações. ${ }^{30}$
Considerando-se que as quedas são influenciadas por fatores intrínsecos e extrínsecos, a variedade de taxas de prevalência encontrada indica a diversidade de situações que envolvem a pessoa idosa que vive em comunidade. . $-6,11,13,15-17,19^{-19}$ A mediana para a prevalência de quedas verificada nessa revisão corrobora os valores apresentados nos textos clássicos sobre prevalência de quedas de idosos. ${ }^{31-35}$

Pode-se dizer que esse evento tem alta prevalência, constituindo um problema multifatorial e que necessita de abordagem por uma equipe multiprofissional. Esses profissionais deverão desenvolver tanto atividades de prontoatendimento ao idoso, como atividades de reabilitação e medidas preventivas, ${ }^{36}$ além de ações de planejamento urbano.

Embora o pronto-atendimento nos serviços de urgência e emergência e a reabilitação ocorra em serviços especializados, o mesmo não ocorre com a prevenção, a qual deve ser amplamente difundida. Evitar o evento de queda é considerada uma conduta de boa prática geriátrica e gerontológica, em hospitais e instituições de longa permanência e comunidade, sendo considerado um dos indicadores de qualidade de serviços para idosos. ${ }^{25}$

Apesar de este estudo ter como limitação a restrição de idiomas selecionados ao inglês, português e espanhol, e ao período de 2000 a 2011, verificou-se que a queda é evento comum na população idosa.

\section{CONCLUSÃO}

A ocorrência de quedas na população idosa que reside na comunidade ainda é alta, com mediana de prevalência de $28,50 \%$.

Apesar de terem sido estudadas várias cidades de diferentes países, ainda são necessários mais dados sobre sua ocorrência, envolvendo populações de regiões geográficas mais amplas, uma vez que se dispõe de apenas três estudos de abrangência nacional (Brasil, Estados Unidos e Nigéria) no período estudado. 


\section{REFERÊNCIAS}

1. Homero Gac E, Pedro Paulo Marín L, Sergio Castro H, Trinidad Hoyl M, Eduardo Valenzuela

A. Caídas en adultos mayores institucionalizados: descripción y evaluación geriátrica. Rev Med Chile 2003;131(8):887-94.

2. Álvarez MN, Rodríguez-Mañas L. Caídas repetidas en el medio residencial. Rev Esp Geriatr Gerontol 2006;41(4):201-6.

3. Stalenhoef PA, Diederiks JP, Knottnerus JA, Kester AD, Crebolder HF. A risk model for the prediction of recurrent falls in community-dwelling elderly: a prospective cohort study. J Clin Epidemiol 2002;55(11):1088-94.

4. Moreno-Martínez NR, Ruiz-Hidalgo D, BurdoyJoaquim E, Vázquez-Mata G. Incidencia y factores explicativos de las caídas en ancianos que viven em la comunidad. Rev Esp Geriatr Gerontol 2005;40(Suppl 1):11-7.

5. Varas-Fabra F, Castro Martín E, Torres LAP, Fernández Fernández MJ, Moral RR, Berge IE. Caídas en ancianos de la comunidad: prevalencia, consecuencias y factores asociados. Aten Primaria 2006;38(8):450-5.

6. Mazo GZ, Liposcki DB, Ananda C, Prevê D. Condições de saúde, incidência de quedas e nível de atividade física dos idosos. Rev Bras Fisioter 2007;11(6):437-42.

7. Blanch MP, Sabaté MQ, Revuelta EA, Blanes RR, Monfort RC. Resultados finales de un estudio de intervención multifactorial y comunitario para la prevención de caídas en ancianos. Aten Primaria 2010;42(4):211-7.

8. Hernández JG, Casas MOP, Pérez MTM, Díaz DB, Lara CM, Coello SD. ¿Podemos desde atención primaria prevenir las caídas en las personas mayores? Aten Primária 2010;42(5):284-91.

9. Gonçalves M, Pereira MP. Muscular coactivation (CA) around the knee reduces power production in elderly women. Arch Gerontol Geriatr 2011;52(3):317-21.

10. Roqueta C, Vázquez O, Jaime E, Castellanos CS, Pastor M, Llobet CC, et al. Evolución de la incidencia de caídas en un centro hospitalario de media y larga estancia. Rev Esp Geriatr Gerontol 2009;44(6):331-4.

11. Formiga F, Ferrer A, Duaso E, Olmedo C, Pujol R. Falls in nonagenarians after 1-year of follow-up: the NonaSantfeliu study. Arch Gerontol Geriatr 2008;46(1):15-23.
12. Di Monaco M, Vallero F, Tappero R, De Lauso L, De Toma E, Cavanna A. Incident falls impair ability to function in hip-fracture survivors: a prospective study of 95 elderly women. Arch Gerontol Geriatr 2009; 48(3):397-400.

13. Halil M, Ulger Z, Cankurtaran M, Shorbagi A, Yavuz BB, Dede D, et al. Falls and the elderly: is there any difference in the developing world? A crosssectional study from Turkey. Arch Gerontol Geriatr 2006;43(3):351-9.

14. Ulus Y, Durmus D, Akyol Y, Terzi Y, Bilgici A, Kuru O. Reliability and validity of the Turkish version of the Falls Efficacy Scale Internatonal (FES-I) in community-dwelling older persons. Arch Gerontol Geriatr 2012;54(3):429-33.

15. Bekibele CO, Gureje O. Fall incidence in a population of elderly persons in Nigeria. Gerontology 2010;56(3):278-83.

16. Chu LW, Chiu AY, Chi I. Falls and subsequent health service utilization in community-dwelling Chinese older adults. Arch Gerontol Geriatr 2008; 46(2):125-35.

17. Fong KNK, Siu AMH, Yeung KA, Cheung SWS, Chan CCH. Falls among the community-living elderly people in Hong Kong: a retrospective study. Hong Kong J Occup Ther 2011;21:33-40.

18. Siqueira FV, Facchini LA, Silveira DS, Piccini RX, Tomasi E, Thumé E, et al. Prevalence of falls in elderly in Brazil: a countrywide analysis. Cad Saude Pública 2011;27(9):1819-26.

19. Cruz HMF, Pimenta CAM, Dellarozza MSG, Braga PE, Lebrão ML, Duarte YAO. Quedas em idosos com dor crônica: prevalência e fatores associados. Rev Dor 2011;12(2):108-14.

20. Siqueira FV, Facchini LA, Piccini RX, Tomasi E, Thumé E, Silveira DS, et al. Prevalência de quedas em idosos e fatores associados. Rev Saude Pública 2007;41(5):749-56.

21. Coimbra AM, Ricci NA, Coimbra IB, Costallat LT. Falls in the elderly of the Family Health Program. Arch Gerontol Geriatr 2010;51(3):317-22.

22. Motta LB, Aguiar AC, Coutinho ESF, Huff G. Prevalência e fatores associados a quedas em idosos em um município do Rio de Janeiro. Rev Bras Geriatr Gerontol 2010;13(1):83-91.

23. Nunes DP, Nakatani AYK, Silveira EA, Bachion MM, Souza MR. Capacidade funcional, condições socioeconômicas e de saúde de idosos atendidos por equipes de Saúde da Família de Goiânia (GO, Brasil). Ciênc Saúde Coletiva 2010;15(6):2887-98. 
24. Stevens JA, Mack KA, Paulozzi LJ, Ballesteros MF. Self-reported falls and fall-related injuries among persons aged $\geq 65$ years--United States, 2006. J Safety Res 2008;39(3):345-9.

25. Santos MMD, Sandoval RA. Análise do risco de quedas em idosos não institucionalizados. EFDeportes. com Rev Digital Buenos Aires [periódico na Internet]. 2009 [acesso em 2 dez 2011];14(136):[aproximadamente 3 p.]. Disponível em: http://www.efdeportes.com/ efd136/analise-do-risco-de-quedas-em-idosos.htm.

26. Gléria PDMP, Sandoval RA. Treinamento funcional como recurso fisioterapêutico para o aprimoramento da força muscular e equilíbrio de idosos. EFDeportes.com Rev Digital Buenos Aires [periódico na Internet]. 2011 [acesso em em 2 dez 2011];16(161):[aproximadamente 4 p.]. Disponível em: http://www.efdeportes.com/efd161/treinamentofuncional-de-idosos.htm.

27. Hulley SB. et al. Delineando a pesquisa clínica. 3 ed. Porto Alegre: Artmed, 2008, 384p.

28. Ribeiro AP, Souza ER, Atie S, Souza AC, Schilithz AO. A influência das quedas na qualidade de vida de idosos. Ciênc Saúde Coletiva 2008; 13(4):1265-73.

29. Aikawa AC, Braccialli LMP, Padula RS. Efeitos das alterações posturais e de equilíbrio estático nas quedas de idosos institucionalizados. Rev Ciênc Med 2006;15(3):189-96.
30. Maia BC, Viana PS, Arantes PMM, Alencar M. Consequencias das quedas em idosos vivendo em comunidade: revisão sistemática. Rev Bras Geriatr Gerontol 2011;14(2):381-93.

31. Blake AJ, Morgan K, Bendall MJ, Dallosso H, Ebrahim SB, et al. Falls by elderly people at home: prevalence and associated factors. Age Ageing 1988;17(6):365-72.

32. Tinetti ME, Speechley M, Ginter SF. Risk factors for falls among elderly persons living in the community. N Engl J Med 1988;319(26):1701-7.

33. Campbell AJ, Borrie MJ, Spears GF, Jackson SL, Brown JS, Fitzgerald JL. Circumstances and consequences of falls experienced by a community population 70 years and over during a prospective study. Age Ageing 1990;19(2):136-41.

34. Svensson ML, Rundgren A, Landahl S. Falls in 84- to 85-year-old people living at home. Accid Anal Prev 1992;24(5):527-37.

35. Luukinen H, Koski K, Hiltunen L, Kivelä SL. Incidence rate of falls in an aged population in northern Finland. J Clin Epidemiol 1994;47(8):843-50.

36. Minaya-Sáiz J, Lozano-Menor A, Guerra RMS. Abordaje multiprofissional de las caídas en un hospital de media estância. Rev Calid Asist 2010;25(2):106-11. 